\title{
Передача энергии электронного возбуждения в массиве квантовых точек CdS на квазидвумерной поверхности
}

\author{
(C) Н.В. Бондарь ${ }^{1}$, М.С. Бродин ${ }^{1}$, Н.А. Матвеевская ${ }^{2}$, Т.Г. Бейник \\ ${ }^{1}$ Институт фризики Национальной академии наук Украины, \\ 03028 Киев, Украина \\ ${ }^{2}$ Институт монокристаллов Национальной академии наук Украины, \\ 61001 Харьков, Украина \\ E-mail: jbond@iop.kiev.ua
}

(Получена 26 июля 2017 г. Принята к печати 5 февраля 2018 г.)

Получены и проанализированы оптические спектры пленок на основе сферических частиц диоксида кремния, покрытых квантовыми точками CdS малого радиуса. Обнаружены большие смещения полос поглощения и фотолюминесценции в зависимости от концентрации квантовых точек, плотности и длины волны накачки. Анализ экспериментальных данных показал, что это обусловлено передачей энергии электронного возбуждения квазичастицами по механизму тунелирования, вызванного сильным взаимодействием между квантовыми точками. Использовав малые плотности накачки и разные длины волн возбуждения, мы получили результаты, позволившие описать распределение квантовых точек CdS по размерам, которое хорошо аппроксимируется функцией Гаусса.

DOI: 10.21883/FTP.2019.02.47098.8697

\section{1. Введение}

В последнее время интенсивно исследуются процессы передачи энергии электронного возбуждения в структурах с разными конфигурациями, в которых доноры и акцепторы принадлежат к органическим веществам или доноры являются полупроводниковыми квантовыми точками (КТ), а акцепторы - органической матрицей. Потребность в этих исследованиях вызвана практической необходимостью, поскольку указанные процессы оказывают существенное влияние на работу светоизлучающих диодов, лазерных элементов, солнечных ячеек, a также фотосинтез и находят свое применение в приборах оптоэлектроники и фотогальваники [1-5]. На сегодня наиболее изученной считается передача энергии при слабом диполь-дипольном взаимодействии между донорами и акцепторами по механизму Фёрстера. Это происходит посредством электромагнитного поля, и эффективность такого процесса может превышать 50\% в зависимости от расстояния между донором и акцептором или спектрального перекрытия между излучением донора и профилем поглощения акцептора $[1,3]$. КТ из полупроводников II-VI, полученные в коллоидном растворе и осажденные на плоскую или выпуклую поверхность, имеют больше преимуществ перед полимерами или красителями при передаче энергии, поскольку являются химически более стабильными, а также благодаря размерному эффекту. В последнее время активно исследуются не массивы одиночных КТ, а так называемые их „строительные блоки“ в связи с созданием структур с плотной упаковкой: двумерные (2D) пленки, трехмерные (3D) сверхрешетки, системы с каскадной передачей энергии (cascaded energy transfer) [2,6-12]. В плотноупакованных массивах возникает сильная связь между КТ, приводящая к туннелированию квазичастиц через барьер, ширина и высота которого регулируются длиной и химической структурой связующего лиганда. Понимание природы этой связи и возможность ее контролировать позволяют улучшить характеристики процессов переноса носителей в приборах на основе этих структур. На сегодня природа сильной связи и передача энергии по этому механизму недостаточно исследованы, и публикации включают лишь несколько структур на основе KT CdS, CdSe и InP [6,7].

В данной работе представлены результаты оптических исследований массивов КТ CdS, выращенных на сферической поверхности $\mathrm{SiO}_{2}$, при различных концентрациях KT, плотностях и длинах волн возбуждения. Полученные данные свидетельствуют о передаче энергии электронного возбуждения между КТ, соединенными короткими молекулярными цепочками полиакриловой кислоты (ПАК) (polyacrylic acid) на поверхности сферических частиц диоксида кремния. Анализ показал, что, вероятнее всего, такой механизм передачи обусловлен туннелированием квазичастиц между КТ в результате сильной связи.

\section{2. Экспериментальные результаты}

Методика изготовления сферических частиц диоксида кремния $\left(\mathrm{SiO}_{2}\right)$ с радиусом $R_{0} \approx 150 \mathrm{Hм}$, квантовых точек $\mathrm{CdS}$, покрытия ими поверхности $\mathrm{SiO}_{2}$ и формирование наночастиц $\mathrm{SiO}_{2} / \mathrm{CdS}$, а также пленок на их основе была описана нами раньше [13]. Схематическое изображение наночастиц приведено на рис. 1, где показана сферическая поверхность $\mathrm{SiO}_{2}$ с двумя КТ CdS разных радиусов, обозначенных как донор $(D)$ и акцептор $(A)$. Поверхность $\mathrm{SiO}_{2}$ модифицирована слоем органических молекул, обеспечивающих ее перезарядку и понижающих поверхностную энергию. Толщина слоя $\sim 1$ нм 


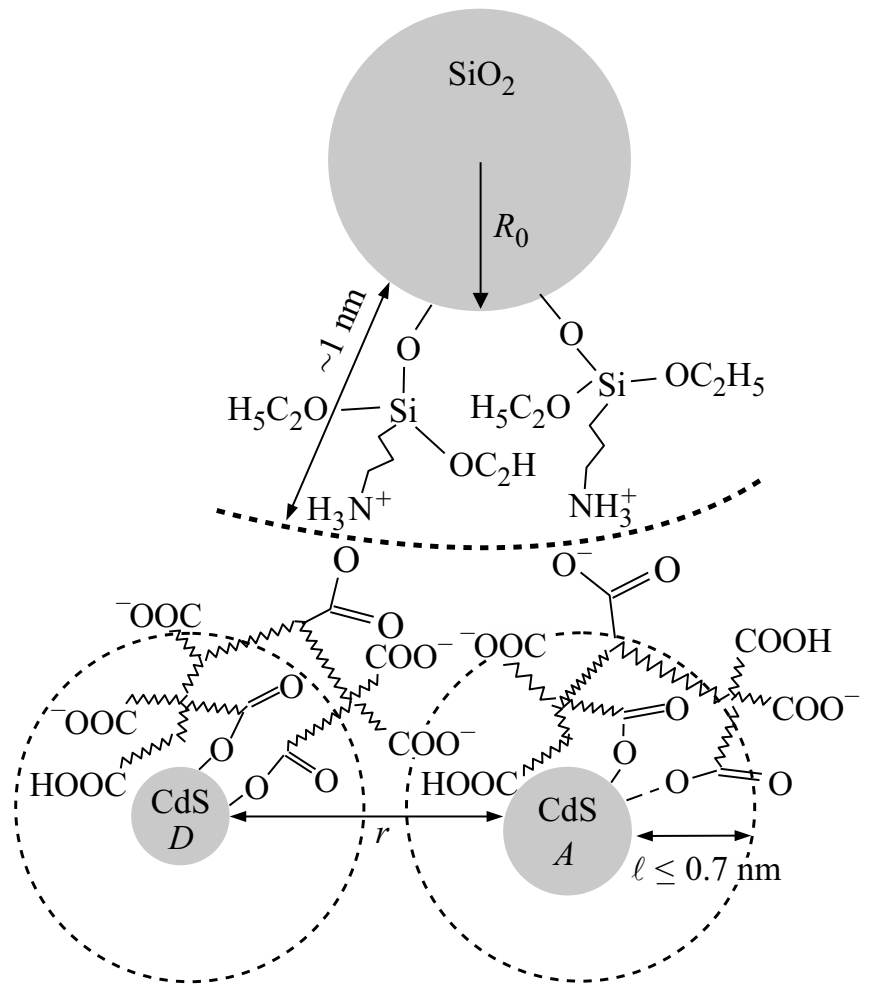

Рис. 1. Схематическое изображение наночастицы: сферическая частица $\mathrm{SiO}_{2}$, модифицированная слоем органических молекул толщиной $\sim 1$ нм, с двумя КT $\mathrm{CdS}$ на ее поверхности, стабилизированных слоем ПАК, толщиной $l \approx 0.7$ нм. Все размеры даны не в пропорции.

предотвращает взаимодействие функциональных групп между собой и устраняет стерические препятствия для равномерного размещения КТ на поверхности $\mathrm{SiO}_{2}$. КТ $\mathrm{CdS}$ получены в водном растворе в присутствии стабилизатора ПАК с молекулярным весом $M_{0} \approx 1800$ г/моль и массовой концентрацией $0.07 \%$. Отметим, что величина $M_{0}$ задает толщину оболочки $(l)$, определяя минимальное расстояние между КТ и в конечном итоге концентрацию последних на сферической поверхности. Для значения $M_{0} \approx 1800$ г/моль рассчитанная толщина оболочки ПАК составила $l<1$ нм. Для проверки мы измерили гидродинамический радиус $\left(R_{g r}\right)$ КТ в коллоидном растворе с помощью динамического рассеяния света (Brookhaven Zetasizer NANO 5S) и получили $R_{g r}=R_{\mathrm{av}}+l \approx 2.41 \mathrm{HM}$, где $R_{\mathrm{av}}-$ средний радиус КТ. Если учесть, что $R_{\mathrm{av}} \approx 1.7$ нм (см. далее), то полученное значение $l \approx 0.7$ нм хорошо согласуется с рассчитанным. $\mathrm{C}$ помощью динамического рассеяния света также была определена дисперсия КТ по размерам в растворе, она составила 10\%.

Нами были изготовлены четыре образца пленок $\mathrm{S} 1-\mathrm{S} 4$ с толщинами $\sim 1$ мкм. Пленки S1 и S2 на основе наночастиц $\mathrm{SiO}_{2} / \mathrm{CdS}$ имели величины покрытия поверхности $\mathrm{SiO}_{2}$ квантовыми точками $\mathrm{CdS} \theta_{1} \approx(0.2 \pm 0.03)$ и $\theta_{2} \approx(0.42 \pm 0.03)$ соответственно. Соотношение кон- центраций чистых частиц $\mathrm{SiO}_{2}$ и КТ в водном растворе составляло $\sim 1 \cdot 10^{-4}$, а количество осажденных КТ регулировалось числом циклов осаждения [13]. Расчет величин $\theta_{1}$ и $\theta_{2}$ дан в следующем разделе. На рис. 2 приведено изображение наночастицы $\mathrm{SiO}_{2} / \mathrm{CdS}$, полученное методом просвечивающей электронной микроскопии (transmission electron microscopy, TEM), где видно равномерное распределение КТ по поверхности $\mathrm{SiO}_{2}$. В качестве тестовых были изготовлены пленки S3 и S4 на основе чистых (непокрытых) сферических частиц $\mathrm{SiO}_{2}$ с $R_{0} \approx 150$ и 410 нм. Все образцы получены методом нанесения наночастиц $\mathrm{SiO}_{2} / \mathrm{CdS}$ или чистых $\mathrm{SiO}_{2}$ в заданной концентрации на подогретую кварцевую подложку, предварительно модифицированную молекулами 3-аминопропилтриэтоксисилана (аminopropyltriethoxysilane, $\left.\mathrm{H}_{2} \mathrm{~N}\left(\mathrm{CH}_{2}\right)_{3} \mathrm{Si}\left(\mathrm{OC}_{2} \mathrm{H}_{5}\right)_{3}\right)$. В качестве пленкообразующего вещества использовалась ПАК с $M_{0}=100000$ г/моль.

На рис. 3 приведены спектры поглощения пленок S1-S4, а также ПАК (на вставке), записанные на спектрометре SHIMADZU 2450 UV-VIS. Результирующие спектры поглощения образцов S1 и S2 (кривые 1 и 2) получены делением исходных спектров S1 и S2 на спектр S3 и нормировкой на соответствующие максимумы при длинах волн $\lambda_{m 1,2}=406$ и 421 нм. Полосы в спектрах S1 и S2 уширены в красную сторону, причиной чего, как мы полагаем, является наличие полосы поглощения в области $\sim 500$ нм (см. спектр 3 на рис. 3), обусловленной поверхностными состояниями $\mathrm{SiO}_{2}$. Для проверки этого была изготовлена пленка $\mathrm{S} 4$, аналогичная $\mathrm{S} 3$, но с $R_{0} \approx 410$ нм спектр поглощения (СП) которой показан на рис. 3 (кривая 4). Мы исходили из предположения, что чем больше радиус сферической

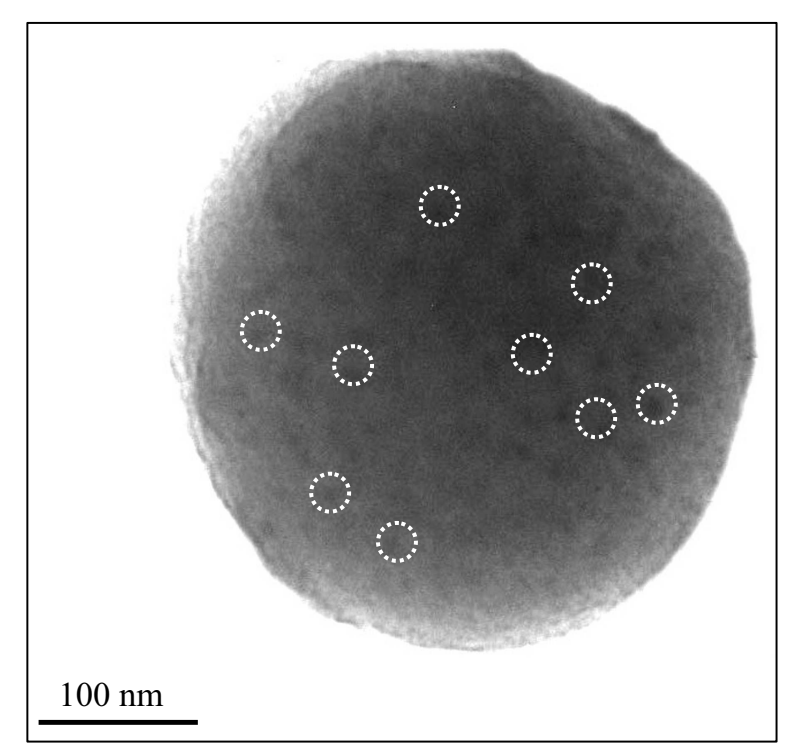

Рис. 2. ТЕМ-изображение сферической частицы $\mathrm{SiO}_{2}$ с КТ CdS на ее поверхности с величиной покрытия $\theta_{1} \sim(0.2 \pm 0.03)$. Кружками отмечены КT CdS. 
частицы $\mathrm{SiO}_{2}$, тем больше ее поверхность приближается к плоскости, и, следовательно, количество поверхностных дефектов должно уменьшиться. Как видно из рис. 3, в области $\sim 500$ нм кривая 4 не имеет каких-либо особенностей, что служит подтверждением нашего предположения. Спектр поглощения ПАК, представленный на вставке к рис. 3, показывает, что молекулы этого стабилизатора также не имеют полос поглощения в указанной области. Спектры фотолюминесценции (ФЛ) исследованных пленок были записаны при температуре $T \approx 300 \mathrm{~K}$. В качестве источников возбуждения ФЛ использовались лазеры с длинами волн $\lambda_{\text {ех }}=325,405$

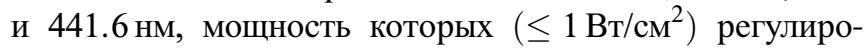

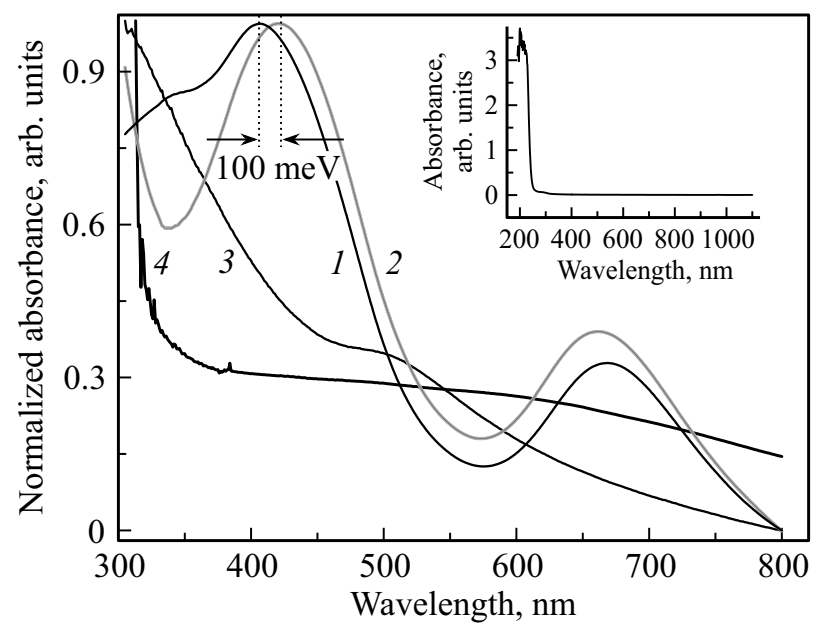

Рис. 3. Спектры поглощения пленок $\mathrm{S} 1-\mathrm{S} 4$ (1-4 соответственно). На вставке - спектр поглощения ПАК.

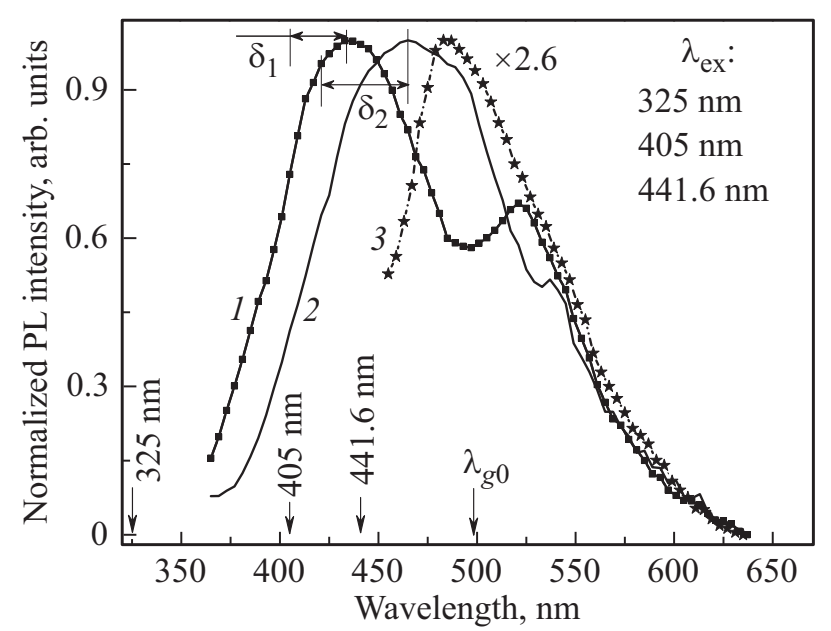

Рис. 4. Спектры фотолюминесценции (PL) пленок S1 (1) и S2 (2) при возбуждении с $\lambda_{\mathrm{ex}}=325$ нм. 3 - одинаковая полоса фотолюминесценции для S1 и $\mathrm{S} 2$, полученная при возбуждении S1 с $\lambda_{\mathrm{ex}}=405$ нм или S2 с $\lambda_{\mathrm{ex}}=441$ нм. $\delta_{1}=190$ и $\delta_{2}=280$ мэВ - величины смещений максимумов полос ФЛ пленок S1 и S2 относительно соответствующих СП. $\lambda_{g 0}=499.8$ нм - длина волны запрещенной зоны объемного CdS.

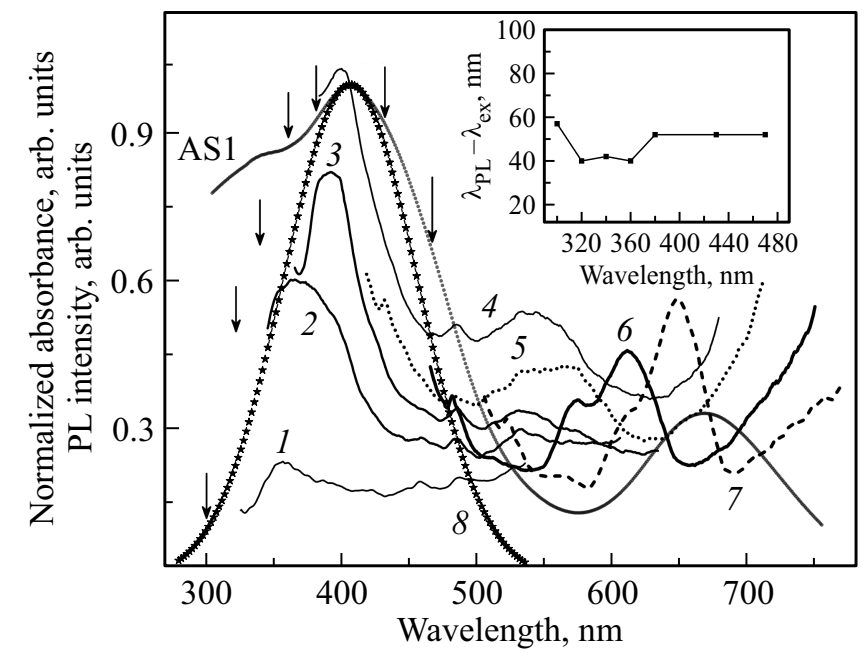

Рис. 5. Полосы фотолюминесценции (PL) (1-7) пленки S1 при возбуждении с длинами волн $\lambda_{\mathrm{ex}}=300,320,340,360,380$, 430 и 470 нм (показаны стрелками). AS1 - полоса поглощения S1 та же, что и на рис. 3. $8-$ функция распеределения КТ по размерам. На вставке - величины смещений длины волны ФЛ $\lambda_{\mathrm{PL}}$ относительно $\lambda_{\mathrm{ex}}$.

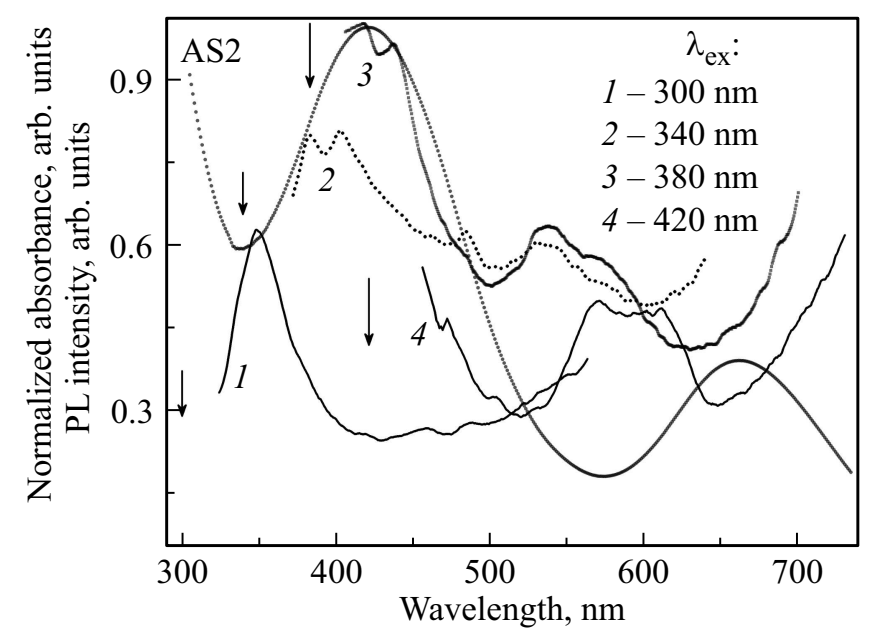

Рис. 6. Полосы фотолюминесценции (PL) (1-4) пленки S2 при возбуждении с $\lambda_{\mathrm{ex}}=300,340,380,420$ нм (показаны стрелками). AS2 - полоса поглощения S2 та же, что и на рис. 3.

валась таким образом, чтобы количество электроннодырочных пар в КТ было примерно одинаковым. Для получения ФЛ пленок при малых плотностях и различных длинах волн возбуждения $\left(\lambda_{\mathrm{ex}}\right)$ использовался спектрометр Perkin Elmer LS-55.

На рис. 4 приведены спектры ФЛ S1 (кривая 1) и S2 (кривая 2), записанные при возбуждении с $\lambda_{\mathrm{ex}}=325$ нм. Видно, что спектры ФЛ обеих пленок состоят из коротко- и длинноволновой компонент. Максимум последней находится в области $\sim 520$ нм, т. е. при энергии меньше запрещенной зоны объемного CdS (соответствующая длина волны $\lambda_{g 0}=499.8$ нм) и, как видно, его 
положение не зависит от концентрации КТ в массиве. Максимумы коротковолновых полос ФЛ пленок S1 и S2 смещены относительно максимумов своих СП соответственно на $\delta_{1} \approx 190$ и $\delta_{2} \approx 280$ мэВ. Наиболее неожиданной оказалась полоса ФЛ 3 на рис. 4, записанная в пленке S1 при возбуждении с $\lambda_{\mathrm{ex}}=405 \mathrm{Hм}$ и в S2 при $\lambda_{\mathrm{ex}}=441.6$ нм, положение которой совпадало в обеих пленках. Кроме этого, ее интенсивность была сопоставимой в обеих пленках и в $\sim 2.6$ раза меньше интенсивности основных полос ФЛ соответствующих пленок. Максимум данной полосы соответствует $483 \mathrm{Hм}<\lambda_{g 0}$, поэтому можно заключить, что она образована квантовыми, а не дефектными или поверхностными состояниями КТ.

На рис. 5 и 6 приведены спектры ФЛ пленок S1 и $\mathrm{S} 2$, записанные при разных $\lambda_{\mathrm{ex}}$ и нормированные на величину наиболее интенсивной из полос спектра: для $\mathrm{S} 1$ это 4, а для $\mathrm{S} 2-3$. В спектрах обеих пленок сначала наблюдается увеличение интенсивности и сужение полос ФЛ с $\lambda_{\mathrm{ex}}$, а затем, при $\lambda_{\mathrm{ex}} \sim \lambda_{m 1,2}$, резкий спад интенсивности ФЛ. При возбуждении в область с $\lambda \geq \lambda_{g 0}$ в обеих пленках наблюдается рост интенсивности ФЛ из глубоких состояний в запрещенной зоне КТ. Полученные результаты позволили описать форму распределения КТ по размерам, которая хорошо аппроксимируется функцией Гаусса (рис. 5, кривая 8). В заключение раздела отметим, что наблюдаемое поведение полос ФЛ в спектрах обеих пленок в зависимости от $\lambda_{\text {ex }}$ и количества КТ можно, вероятнее всего, объяснить передачей энергии электронного возбуждения между КТ при сильной их связи, что обсуждается далее.

\section{3. Обсуждение полученных результатов}

\section{1. Квантовые точки на квазидвумерной поверхности}

Передача энергии электронного возбуждения между КТ зависит от значения $\theta$, определяющего степень покрытия поверхности сферических частиц $\mathrm{SiO}_{2}$. При минимальном покрытии $\left(\theta_{\min }\right)$ взаимодействие между КТ практически отсутствует, поэтому форма полосы ФЛ воспроизводит их форму распределения по размерам. На первый взгляд найти $\theta$ просто, если считать КТ твердыми сферическими частицами, плотно покрывающими поверхность сферы. Тогда $\theta=N / N_{0}$, где $N$ - число подсчитанных КТ на поверхности, а $N_{0}$ - максимальное их количество, которое можно получить, разместив их на сферической поверхности в форме гексагональной плотной упаковки [14]:

$$
N_{0} \approx \frac{2 \pi \sqrt{3}}{3} B^{2}\left[\left(1+B^{-1}\right)^{2}-0.773 B^{-2}\right],
$$

где $B=R_{0} / R_{g r}$. В отличие от выражения в [14], здесь в квадратных скобках добавлен член для выполнения асимптотики: $N_{0} \rightarrow 12$ при $B \rightarrow 1$. Если $B \gg 1$,
$N_{0} \sim 2 \pi B^{2} / \sqrt{3}$. Однако при случайном заполнении поверхности сферы плотная упаковка из КТ не образуется, поэтому значение $\theta$ будет заниженным. Для получения реальных значений необходимо принять во внимание, что осаждение КТ на поверхность сферы является случайным процессом, для описания которого часто используют RSA-модель (random sequential absorption) [15]. В этой модели предполагается, что после осаждения КТ на поверхность сферы она „прилипает“ к ней за счет электростатического взаимодействия, становясь неподвижной. Процесс осаждения КТ на поверхность прекращается при достижения так называемой блокадной границы (jamming limit), т. е образования квазимонослоя КТ при $\theta_{m}=\theta_{0}\left(1+R_{g r} / R_{0}\right)^{2}$, где $\theta_{0}=0.547-$ блокадная граница для плоской поверхности. Следовательно, доступная для КТ площадь поверхности сферы составляет $S_{0}=4 \pi R_{0}^{2} \theta_{m}$, а максимальное число КТ, которое можно разместить на ней, $N_{m}=S / \pi R_{g r}^{2}$. Если $R_{0} \gg R_{g r}$, то $N_{m} \approx 4 \theta_{0}\left(R_{0} / R_{g r}\right)^{2}$. Просканировав поверхность частиц в пленках $\mathrm{S} 1$ и $\mathrm{S} 2$ и подсчитав количество КТ $N_{1,2}$, мы получили величины покрытия $\theta_{1,2}=N_{1,2} / N_{m}$. На квазидвумерной поверхности сферы может образоваться перколяционный кластер экситонов при $\theta_{*} \sim \theta_{m} / 2$ с вероятностью $\sim 1 / 2$, что для $2 \mathrm{D}$ систем считается нижней границей перколяции. Однако в наших пленках $\theta_{1,2}<\theta_{*}$, поэтому можно считать, что экситоны в обоих образцах находятся ниже порога перколяции.

\section{2. Передача энергии возбуждения между КT при сильной связи}

При сильном взаимодействии передача энергии электронного возбуждения между КТ также зависит и от $R_{\mathrm{av}}$. Для его нахождения используем метод эффективной массы, выбрав одинаковые значения потенциальных ям для электронов и тяжелых дырок: $V_{e}=V_{h h} \approx 4.5$ эВ. Они обеспечиваются широкозонными матрицами ПАК и $\mathrm{SiO}_{2}$, поглощение которых начинается в области $\lambda<300$ нм, что видно из рис. 3. Как показали авторы [16], для эффективных масс электронов и дырок в $\mathrm{CdS}$ значение потенциала $V \geq 2.5$ эВ уже является достаточным, чтобы считать потенциальные ямы бесконечно глубокими. Кроме метода эффективной массы для определения $R_{\mathrm{av}}$ часто используют выражение, полученное авторами [17], которое, несмотря на то что было получено эмпирическим путем, дает хорошие результаты. Для КT CdS оно имеет вид

$$
\begin{aligned}
D^{*}=2 R_{\mathrm{av}}=( & \left.-6.6521 \cdot 10^{-8}\right) \lambda^{3}+\left(1.9557 \cdot 10^{-4}\right) \lambda^{2} \\
& -\left(9.2352 \cdot 10^{-2}\right) \lambda+13.29
\end{aligned}
$$

где $\lambda$ - длина волны экситонного перехода $1 S$. Использовав положение максимума экситонного перехода спектра поглощения пленки S1 на рис. 3, соответствующее основному переходу $\left(1 S_{e}-1 S_{3 / 2 h}\right)$, получаем значения $R_{\mathrm{av}} \approx 1.76$ и $1.84 \mathrm{HM}$, которые хорошо согласуются с 
$R_{g r}$ с учетом толщины оболочки ПАК $l \approx 0.7$ нм. Теперь отметим, что только при $\theta \leq \theta_{\min } \mathrm{KT} \mathrm{CdS} \mathrm{можно} \mathrm{считать}$ изолированными на поверхности сферы, вероятность обмена элементарными возбуждениями между которыми незначительная. В этом случае форма полосы ФЛ воспроизводит форму распределения КТ по размерам, а положение максимума спектра поглощения совпадает с длиной волны излучения КТ с $R_{\mathrm{av}}$. При $\theta \geq \theta_{\min }$ в результате формирования кластеров КТ и уменьшения энергии размерного квантования максимум спектра поглощения сместится и $R_{\mathrm{av}}$ увеличится. Точно определить $\theta_{\min }$ сложно, но, по нашим оценкам [13], оно близко к $\theta_{1}$.

Для возможности наблюдать передачу энергии в массиве КТ необходимо выполнение нескольких условий. Во-первых, одни КТ в массиве должны быть донорами с бо́льшей энергией экситонов, а другие акцепторами. В наших пленках это обеспечивается $\sim 10 \%$-й дисперсией размеров КТ. Во-вторых, расстояние между соседними КТ должно быть малым. Высота и ширина барьера между КT $\mathrm{CdS}$ как потенциальными ямами регулируется величиной $2 l \approx 1.4$ нм. Значение $R_{\text {av }} \ll a_{x}$, где $a_{x} \approx 3$ нм - боровский радиус экситона в объемном $\mathrm{CdS}$, обеспечивает сильное сжатие волновой функции экситонов в КТ и ее проникновение за пределы последних. Для оценки константы затухания волновой функции $(\beta)$ за пределами КТ $\mathrm{CdS}$ воспользуемся ВКБ-приближением [7]: $\beta \sim\left(2 m_{e}^{*} \Delta E / \hbar^{2}\right)^{1 / 2}$, где $\Delta E \approx 3$ эВ - высота барьера на границе КТ-ПАК, $m_{e}^{*} \approx 0.5 m_{0}-$ эффективная масса электрона в ПАК. В результате получаем: $\beta \approx 0.63 \AA^{-1}$, что меньше аналогичной величины $\left(\sim 1.1 \AA^{-1}\right)$ в таких часто используемых лигандах, как хлориды, тиоцианаты, сульфиды, тетрафлюоробораты, гидразин и пиридин (chloride, thiocyanate, sulfide, tetrafluoroborate, hydrazine, pyridine) [12].

Сравнение положения полос спектров поглощения S1 и S2 на рис. 3 показывает большое красное смещение между ними, составляющее $\sim 100$ мэВ. Величина больше этой, $\sim 170$ мэВ, была получена только в массиве KT CdTe с $R<2.5$ нм [12]. Величины смещений между максимумами спектра поглощения и ФЛ пленок S1 и $\mathrm{S} 2$ составляют $\delta_{1} \approx 190$ и $\delta_{2} \approx 280$ мэВ. Эти значения нельзя объяснить стоксовым смещением между поглощением и ФЛ, часто наблюдаемым в КТ полупроводников II-VI. Одну из причин появления стоксова смещения связывают с тонкой структурой экситона (темные и светлые состояния) или с поверхностными и дефектными состояниями КТ [16]. Однако увеличение смещения полосы ФЛ, $\delta_{2}$, с ростом числа КТ $\mathrm{CdS}$ доказывает, что, вероятно, в даном случае это не так, а дефектные и поверхностные состояния КТ, о которых речь далее, находятся в области $\lambda>\lambda_{g 0}$. Другая возможная причина - укрупнение КТ за счет их слипания на поверхности $\mathrm{SiO}_{2}$ во время осаждения. Однако, как показывают данные ТЕМ, этого не происходит, поскольку КТ хорошо застабилизированы слоем ПАК. Наблюдаемое красное смещение полос поглощения в наших пленках можно объяснить гибридизацией зонных орбиталей электронов $\left(1 S_{e}\right)$ и дырок $\left(1 S_{3 / 2 h}\right)$ в результате сильного электронного взаимодействия $[4,6-10]$. Это взаимодействие увеличивает делокализацию волновой функции пары $e-h$, уменьшая энергию размерного квантования при переходе с донора на акцептор. Сильное взаимодействие между КТ, приводящее к туннелированию (квантовым резонансам), препятствует формированию диполей в центре КТ, поэтому диполь-дипольное взаимодействие не играет существенной роли уже при $R_{\mathrm{av}} \approx 2 l$ [6]. Эффективность передачи энергии в массиве КТ растет с увеличением их концентрации, когда начинают формироваться кластеры КТ, как это следует из рис. 4 (спектр 2).

Таким образом, качественно полученные результаты можно объяснить, допустив, что энергия взаимодействия между KT $\mathrm{CdS}$ зависит от $R_{\mathrm{av}}$ при минимальном расстоянии между их поверхностями $\sim 2 l$. Вслед за авторами [10] определим эту энергию как уменьшение энергии размерного квантования самого нижнего экситонного перехода в КТ, которая в нашем случае составляет $\sim 100$ мэВ. Энергия взаимодействия определяется интегралом передачи (transfer integral) через перекрытие орбиталей [1]: $I(R)=I_{0}\left(R_{\mathrm{av}}\right) \exp \left(D_{0} \beta / 2 R_{\mathrm{av}}\right) \exp (-D \beta / 2 R)$, где $I_{0}\left(R_{\mathrm{av}}\right) \sim \Delta E_{1 S}\left(R_{\mathrm{av}}\right)-$ энергия размерного квантования $1 S$-экситонного перехода, $D_{0}-$ расстояние между соседними КТ, при котором перекрытие орбиталей максимальное. В результате получаем: $I(R) \approx 100$ мэВ при $R_{\mathrm{av}} \approx 1.76 \mathrm{Hм}$ и $l \approx 0.78$ нм, что близко к значениям, полученным в эксперименте. Значения $\Delta E_{1 s}\left(R_{\mathrm{av}}\right)$ и $I(R)$ уменьшаются с ростом $R$, и волновые функции экситонов локализуются в КТ. Как результат гибридизация орбиталей электронов и дырок быстро падает. В связи с этим возникает вопрос: как далеко может сместиться в красную сторону максимум полосы ФЛ при дальнейшем увеличении числа КТ в массиве? Диффузия экситонов в массиве должна обеспечить их захват в KT CdS с наибольшими $R$. Однако при туннелировании из меньших КТ в бо́льшие уменьшается энергия размерного квантования экситонов и их волновая функция все больше локализуется в КТ, слабо проникая за их пределы. Поэтому диффузия экситонов продолжится

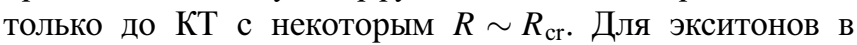
КТ с $R>R_{\text {сг }}$ взаимодействие исчезнет и экситоны в них будут затухать излучательно, как в коллоидном растворе. Время излучательной рекомбинации таких экситонов сравнивается с временем туннельного прыжка. Обнаружить экспериментально КТ с $R_{\text {cr }}$ можно и без увеличения их количества в массиве. Для этого следует при данной концентрации возбуждать КТ с радиусами, находящимися в пике функции распределения или больше, т.е. с $R \geq R_{\mathrm{av}}$. Положение полосы $Ф$ Л, полученной при этом, будет обусловлено КТ с $R_{\text {cr }}$ и ее положение не должно зависеть от $\lambda_{\text {ex }}$.

На наш взгляд, полоса 3 на рис. 4 отвечает этому требованию, поскольку ее спектральное положение не 


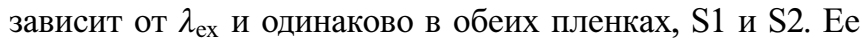
форма асимметрична, с резким коротковолновым краем, a ее длинноволновый край практически совпадает по форме с краем спектров 1 и 2 на рис. 4. Максимум этой полосы отвечает $1 S$-экситонному переходу в КТ $\mathrm{CdS}$ с $R_{\mathrm{cr}} \approx 3.71 \mathrm{Hм,} \mathrm{при} \mathrm{этом} R_{\mathrm{av}} / R_{\mathrm{cr}} \approx 1 / 2$. Таким образом, если вырастить массив КТ с $R_{\mathrm{av}} \approx R_{\mathrm{cr}}$, то диффузия экситонов в нем будет ограниченной только КТ с $R<R_{\text {av }}$. На наш взгляд, именно такую ситуацию анализировали авторы [18] на примере массива КТ $\mathrm{Si}$ в матрице $\mathrm{SiO}_{2}$. Поэтому, создавая массивы КТ с разными $R$ и разной длиной лиганда, можно наблюдать смену типа взаимодействия от туннельного до слабого, диполь-дипольного, и смену механизма передачи энергии возбуждения (по Фёрстеру). Такая смена типа взаимодействия и механизма отмечалась авторами [10] в массиве KT CdTe с $R_{\mathrm{av}} \approx 5.1 \mathrm{Hм}$.

Возбуждение пленок с помощью лазерной накачки создает в КТ большое число экситонов, диффузия которых приводит к смещению между полосами СП и ФЛ. Использование же ламповой накачки позволяет значительно уменьшить их количество, поэтому лишь малая доля КТ будет в возбужденном состоянии. Наиболее эффективно возбуждаются КТ, для которых длина волны, соответствующая оптической щели экситонов,

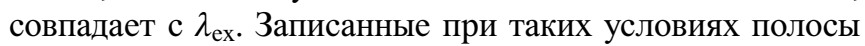
ФЛ пленок S1 и S2 показаны на рис. 5 и 6. Похожие исследования недавно провели авторы [19], но для обратного процесса - передачи энергии возбуждения из KT CdS в органическую матрицу. Характерной чертой спектров ФЛ с ростом $\lambda_{\mathrm{ex}}$ является увеличение интенсивности ФЛ и смещение ее максимума в сторону $\lambda_{m 1,2}$. При $\lambda_{\mathrm{ex}} \geq \lambda_{m 1,2}$, происходит красный сдвиг и резкое уменьшение интенсивности полос ФЛ, после чего в спектре остаются только полосы ФЛ из области поверхностных и дефектных состояний КТ с $\lambda \geq \lambda_{g 0}$. Отметим, что похожее смещение полос ФЛ с $\lambda_{\mathrm{ex}}$ отмечали и авторы $[20,21]$ в КТ 3C-SiC, выращенных в желатиновой матрице, однако должного объяснения этому явлению не было дано.

Уменьшение плотности накачки позволяет уменьшить сдвиг $\Delta \lambda=\lambda_{\mathrm{PL}}-\lambda_{\mathrm{ex}}$ максимума полосы ФЛ при $\lambda_{\mathrm{PL}}$ относительно $\lambda_{\mathrm{ex}}$ (см. вставку к рис. 5). Это дает возможность зондировать КТ и описать их функцию распределения по размерам. Увеличивая $\lambda_{\mathrm{ex}}$, мы наблюдали рост интенсивности ФЛ в пленках S1 и S2. При $\lambda_{\mathrm{ex}}=360$ нм полоса ФЛ резко сужается (рис. 5, полоca 4), поскольку возбуждается большое количество КТ с узким распределением по размерам возле максимума СП. Дальнейшее увеличение $\lambda_{\mathrm{ex}}$ до 380 нм приводит к возбуждению максимального числа КТ-доноров, находящихся в максимуме функции распределения. Количество КТ-акцепторов при этом значительно меньше, поэтому интенсивность полос ФЛ 5 в пленке S1 и 4 в $\mathrm{S} 2$ резко падает. Отметим, что максимумы этих полос совпадают с полосами ФЛ, записанными при лазерном возбуждении с $\lambda_{\mathrm{ex}}=325 \mathrm{нм}$ (рис. 4). Можно отметить два значения $\lambda_{\mathrm{ex}}=325,405$ нм, при которых происходит резкое нарастание и падение интенсивности полосы ФЛ. Если разность этих значений принять за примерную полуширину функции распределения КТ по размерам, то ее можно аппроксимировать функцией Гаусса с дисперсией $\sigma \approx 35$ нм (полоса 8 на рис. 5). Это распределение действительно у́же, чем спектр поглощения пленки S1, уширеннного за счет вклада поверхностных и глубоких дефектных состояний КТ.

В завершение скажем несколько слов о дефектных и поверхностных состояниях, их детальное обсуждение не входит в нашу задачу. Спектр ФЛ сферических частиц $\mathrm{SiO}_{2}$ без КТ не имеет полосы излучения в области $\sim 520$ нм, однако она там появляется при осаждении KT CdS на поверхность частиц (рис. 4-6). Возможная причина заключается в том, что, как и в случае с КТ Si на поверхности $\mathrm{SiO}_{2}$ [22], электрон (дырка) или экситон из КТ $\mathrm{CdS}$ захватываются на двойную связь $\mathrm{Si}=\mathrm{O}$ на поверхности сферы, образуя уровень, соответствующий $\lambda \approx 520$ нм. Глубокие состояния в КТ формируют полосу ФЛ в области 650-700 нм, которая наблюдается в КТ $\mathrm{CdS}$ с различными стабилизаторами, помещенных в разные органические растворители: $\mathrm{C}_{3} \mathrm{H}_{8} \mathrm{O}, \mathrm{C}_{8} \mathrm{H}_{17} \mathrm{OH}$, $\mathrm{CCl}_{4}, \mathrm{C}_{6} \mathrm{H}_{14}, \mathrm{CHCl}_{3}, \mathrm{CH}_{2} \mathrm{Cl}_{2}$ или $\mathrm{C}_{3} \mathrm{H}_{6} \mathrm{O}$ [23]. Таким образом, к перечисленным стабилизаторам можно добавить и ПАК, поскольку она создает глубокие состояния в $\mathrm{KT} \mathrm{CdS}$, формирующие не только полосу ФЛ, но и спектр поглощения.

\section{4. Заключение}

Таким образом, в работе получены и проанализированы спектры пленок на основе KT CdS, выращенных в коллоидном расстворе и осажденных на поверхность сферических частиц $\mathrm{SiO}_{2}$ с разной величиной покрытия последних. Обнаружено красное смещение между СП и ФЛ, зависящее от концентрации КТ, интенсивности и длины волны накачки. Полученные результаты подтверждают эффективную передачу энергии электронного возбуждения в массиве КТ через механизм туннелирования носителей в результате сильной связи между КТ. Использование различных длин волн накачки позволило просканировать массив КТ и описать их распределение по размерам, которое хорошо аппроксимируется функцией Гаусса. Сделан вывод, что должны существовать КТ с таким критическим радиусом $R_{\text {cr }}$, что для всех КТ с $R / R_{\text {cr }}>1$ взаимодействие и передача энергии между ними будут отсутствовать. Помимо этого обнаружено, что ПАК, как и другие стабилизаторы, формирует полосу ФЛ поверхностных и глубоких дефектных состояний, однако их влияние на квантовые (внутренние) состояния KT CdS незначительное.

Работа выполнена в рамках целевой комплексной программы фундаментальных исследований НАН Украины „Фундаментальные проблемы создания новых наноматериалов и нанотехнологий“, проект НАНО № 2-16-Н. 


\section{Список литературы}

[1] F. Remacle, R.D. Levine. ChemPhysChem., 2, 20 (2001).

[2] N. Kholmicheva, P. Moroz, H. Eckard, G. Jensen, M. Zamkov. ACS Energy Lett., 2, 154 (2017).

[3] N. Hildebrandt, C.M. Spillmann, W.R. Algar, T. Pons, M.H. Stewart, E. Oh, K. Susumu, S.A. Díaz, J.B. Delehanty, I.L. Medintz. Chem. Rev., 117, 536 (2016).

[4] A.P. Litvin, E.V. Ushakova, P.S. Parfenov, A.V. Fedorov, A.V. Baranov. J. Phys. Chem. C, 118, (6531) (2014).

[5] А.А. Зарубанов, К.С. Журавлев, Т.А. Дуда, А.В. Окотруб. Письма ЖЭТФ, 95, 403 (2012).

[6] DaeGwi Kim, S. Tomita, K. Ohshiro, T. Watanabe, T. Sakai, I-Ya. Chang, K. Hyeon-Deuk. Nano Lett., 15, 4343 (2015).

[7] Tobias Hanrath. J. Vac. Sci. Technol. A, 30, 030802 (2012).

[8] Fan Xu, L.F. Gerlein, Xin Ma, Ch.R. Haughn, M.F. Doty, S.G. Cloutier. Matterials, 8, 1858 (2015).

[9] C.R. Kagan, C.B. Murray, M.G. Bawendi. Phys. Rev. B, 54, 8633 (1996).

[10] R. Koole, P. Liljeroth, C. de Mello Donega, D. Vanmaekelbergh, A. Meijerink. J. Am. Chem. Soc., 128, 10436 (2006).

[11] H. Do1lefeld, H. Weller, A. Eychmuller. Nano Lett., 1, 267 (2001).

[12] K.N. Lawrence, M.A. Johnson, S. Dolai, A. Kumbhar, R. Sardar. Nanoscale, 7, 11667 (2015).

[13] Н.В. Бондарь, М.С. Бродин, А.М. Бродин, Н.А. Матвеевская. ФТП, 50 (3), 369 (2016).

[14] M. Alonso, M. Satoh, K. Miyanami. Powder Technol., 62, 35 (1990).

[15] Z. Adamzhyk. Particles at Interfaces: Interactions, Deposition, Structure (Academic Press, Amsterdam-BostonHeidelberg, 2006).

[16] J.L. Marın, R. Riera, S.A. Cruz. J. Phys. Condens. Matter, 10, 1349 (1998).

[17] W. William Yu, Lianhua Qu, W. Guo, Xiaogang Peng. Chem. Matt., 15, 2854 (2003).

[18] В.А. Беляков, К.В. Сидоренко, А.А. Конаков, Н.В. Курова, В.А. Бурдов. ФТП, 46 (12), 1613 (2012).

[19] А.А. Зарубанов, В.Ф. Плюснин, К.С. Журавлев. ФТП, $51(5), 605$ (2017).

[20] J. Wang, S.J. Xiong, X.L. Wu, T.H. Li, P.K. Chu. Nano Lett., 10, 1466 (2010).

[21] J. Zhu, Z. Liu, X.L. Wu, L.L. Xu, W.C. Zhang, P.K. Chu. Nanotechnol., 18, 365603 (2007)

[22] M.V. Wolkin, J. Jorne, P.M. Fauchet. Phys. Rev. Lett., 82, 197 (1999).

[23] U. Resch, A. Eychmiiller, M. Haase, H. Weller. Langmuir, 8, 2215 (1992).

Редактор Л.В. Шаронова

\section{Electronic excitation energy transfer in arrays of quantum CdS dots on a quasi-two-dimensional surface}

\author{
N.V. Bondar ${ }^{1}$, M.S. Brodyn ${ }^{1}$, \\ N.A. Matveevskaya ${ }^{2}$, T.Beynik ${ }^{2}$ \\ ${ }^{1}$ Institute of Physics, \\ National Academy of Sciences of Ukraine, \\ 03028 Kiev, Ukraine \\ 2 Institute for Single Crystals, \\ National Academy of Sciences of Ukraine, \\ 61001 Kharkov, Ukraine
}

\begin{abstract}
Optical spectra of films based on silica spheres coated with small-radius CdS quantum dots were obtained and analyzed. large shifts of absorption and photoluminescence bands as a function of the concentration of quantum dots, the density, and the pump wavelength were detected. An analysis of the experimental data has shown that this is due to the transfer of the electron excitation energy by quasiparticles according to the tunneling mechanism caused by a strong interaction between quantum dots. Using small pump densities and different excitation wavelengths, we obtained the results which enabled us to describe the distribution of CdS quantum dots in size, which was well approximated by the Gaussian function.
\end{abstract}

